\title{
Estimating the Risk of Satellite Collisions in Densely Populated Orbit Shells
}

\author{
William Lee ${ }^{1,2} \dagger$, Paul Martin $^{3}$ and Ann Smith ${ }^{1}$ \\ ${ }^{1}$ University of Huddersfield, Huddersfield, UK \\ ${ }^{2}$ University of Limerick, Limerick, Ireland \\ ${ }^{3}$ University of Leeds, Leeds, UK \\ (Communicated to MIIR on 15 November 2021)
}

Study Group: ESGI 162, Leeds, 20-24 July 2020

Communicated by: J.A. Ward

Industrial Partner: Transfinite Systems

Presenter: John Parker.

Team Members: Giancarlo A. Antonucci, University of Oxford; Georgia Brennan, University of Oxford; Yoana Grudeva, University of Portsmouth; Alfred Kume, University of Kent; William Lee, Univesity of Huddersfield; Paul Martin, University of Leeds; Nur Anisah Mohamed, Universiti Malaya; Colin Please, University of Oxford; Joe Roberts, University of Oxford; Ann Smith, University of Huddersfield; Tamsin Spelman, Cambridge University.

Industrial Sector: Aerospace.

Tools: Probability, Asymptotics, Numerical Analysis.

Key Words: Collision probability. Integral approximation.

MSC2020 Codes: 70F16 Collisions in celestial mechanics, regularization; 70F35 Collision of rigid or pseudo-rigid bodies; 41A60 Asymptotic approximations, asymptotic expansions (steepest descent, etc.).

$\dagger$ Corresponding Author: w.lee@hud.ac.uk 


\section{Summary}

Low Earth Orbit is becoming crowded with satellites. Updating estimates of collision probabilities is important as new deployments are authorised but is difficult because only limited information is given.

This report investigates developing analytic estimates of collision probabilities. A survey of approaches reported in the literature is carried out. A collision involving a satellite from the Iridium cluster is reviewed. A simple analytic expression for the collision probability between two satellites is derived using the smallness of several dimensionless ratios appearing in the problem. Single collision probabilities are then extended to orbital planes populated by $n$ satellites with the aim of finding the optimal point at which to traverse such an orbit.

This report demonstrates that analytic estimates relevant to the problem can be made. Further work should focus on: making these estimates rigorous by using a formal asymptotic approach, considering multiple orbital planes and introducing time dependence. 


\section{Introduction}

\subsection{Statement of the problem}

Low Earth Orbit (LEO) describes orbits at heights between 160km and 1000km above the Earth's surface. This volume is getting crowded and it is estimated that there will eventually be 70,000 satellites in Low Earth Orbit. As some orbit shells (heights) are preferred satellites are more densely packed than would be expected if satellites were uniformly distributed.

There is a real risk that collisions between satellites will occur. This was highlighted by the 2009 collision between the Iridium-33 and Kosmos-2251 satellites. This is currently mitigated against by regular assessment of current and future satellite positions by radar tracking and orbital modelling. However, these both involve a degree of uncertainty. Positions cannot be measured exactly and orbits cannot be predicted perfectly. Astronomical forces such as gravitational tugs by the Sun and Moon can be adjusted for, but satellites are not inert and may regularly perform station keeping manoeuvres which are difficult to predict. (For example, an earth observation satellite may regularly adjust its orbit to stay close to the terminator.) When the predicted probability that two satellites will collide crosses a threshold the operators of those two satellites coordinate an avoidance manoeuvre.

The problem of assessing collision risk in the case where we know the (approximate) orbital elements of the satellites involved is already complex. However, in considering new satellite deployments even these data are not available. As a concrete example consider assessing the risks involved in the planned deployment by SpaceX of 1584 Starlink satellites in a $550 \mathrm{~km}$ orbit. This deployment already has regulatory approval but the only information available is the number of satellites, the height of the orbit shell, and that the satellites will be evenly spread and have the same orbital inclination (and it may be that some satellites will be kept above this shell in reserve).

The question this report addresses is: What estimates can we make about the increase in collision probability due a new deployment about which limited information is known? It is anticipated that this will mostly be due to potential collisions between satellites previously deployed and the satellites forming the new deployment as sketched in Fig. 1. Answering this question will be important in determining the point at which high probabilities of collisions occur so frequently that the current method of addressing these is no longer viable.

\subsection{Outline}

The rest of the report is organised as follows. In section 2 we consider the background to this work. We discuss the two line element sets which are used to describe orbits and the deterministic aspects of their drift over time. We also review the literature that was consulted in putting together this report which contains the models that were adapted to estimate collision probabilities. Section 3 summarises what is known about the collision between the Iridium-33 satellites and the Kosmos-2251 satellite and describes how a historical record of orbital elements was used to estimate uncertainties in the position of a satellite. We discuss a simple model for estimating the probability of collision between 


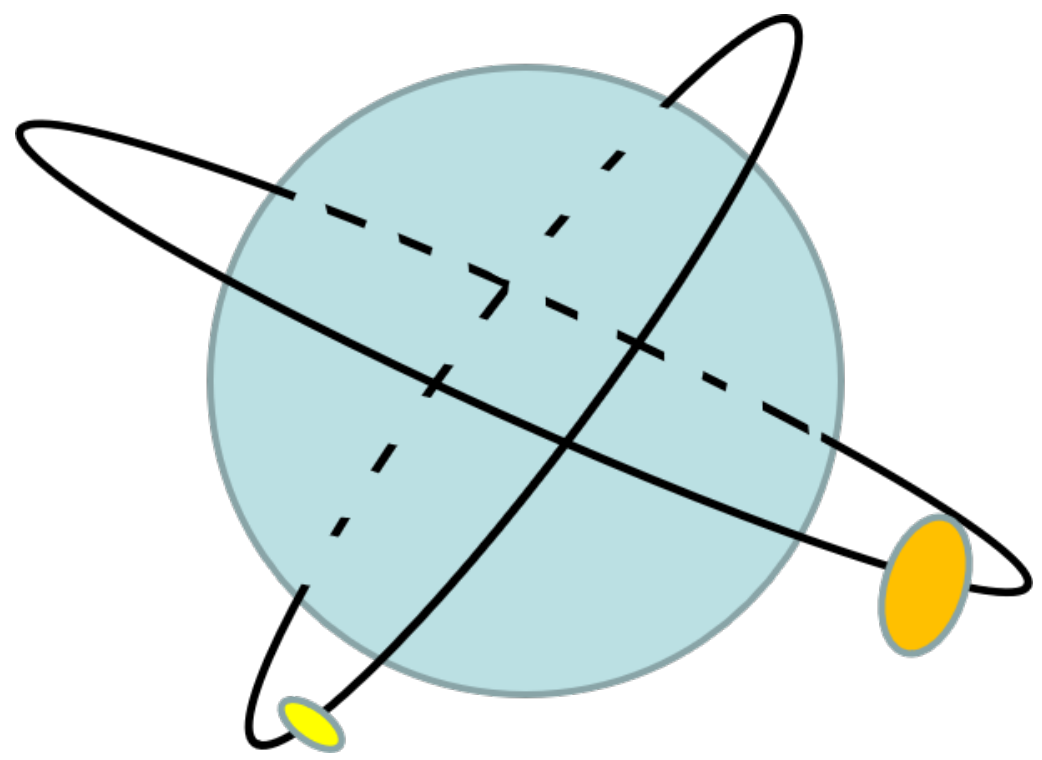

Figure 1. A new satellite deployment will be at most risk of colliding with satellites previously deployed as these orbits are most likely to cross.

two satellites with orbits at significantly different inclinations in section 4 . In section 5 the scaling up of this estimate to two or more interacting constellations of satellites is discussed. Conclusions and recommendations are given in section 6. An appendix gives a detailed discussion of the mathematics involved in combining probability distributions. 


\section{Background}

In this section we describe how satellite positions are described using two line element sets and review analytic techniques used to estimate collision probabilities.

\subsection{Two Line Element Sets}

Every 15 hours the position of each satellite in low Earth orbit is measured. These measurements are summarised in a two line element set an example of which is given here:

$\begin{array}{llllllll}124946 \mathrm{U} & 97051 \mathrm{C} & 09040.36587205 & .00000121 & 00000-0 & 36194-4 & 0 & 4723\end{array}$

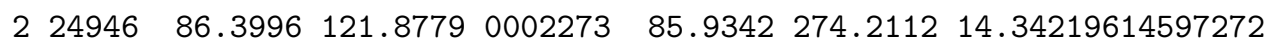

The elements given do not just describe the current orbit of the object but also contain parameters describing drift that can be used by Simplified Perturbations Models (SGP) to predict future orbits. Two line element data may be downloaded, for example from www. celestrak.com.

Below, in section 3 we discuss the limitations of these measurements, which were not able to predict the collision between a satellite within the Iridium constellation and the Kosmos-2251 satellite. We also use historical data from an NOAA satellite to carry out our own estimates of uncertainties in positions.

\subsection{Literature consulted}

A literature survey was conducted investigating analytic and semianalytic tools for estimating the probability of satellite collisions in the cases where orbital elements are at least roughly known.

Campbell (2004) developed an analytic technique for estimating collision probabilities with the aim of using it as a component of an autonomous collision avoidance system [1]. Campbell's simplified collision prediction algorithm assumed that the uncertainty in position (due to initial errors in location, sensor errors and drift) can be bounded by an ellipsoid. The method combines estimates based on (1) distances between probability ellipsoids, (2) overlap of ellipsoids and (3) convolution integrals.

In 2009 Chan carried out a risk assessment of the probability the International Space Station (ISS) would collide with space debris [2]. Chan used an analytic method, the Method of Equivalent Cross Sectional Area (MECSA), which involved considering each section of the ISS separately. This allowed the then standard assumption of a spherical cross section to be relaxed and a more realistic cross section to be simulated. Chan reported agreement to within $0.1 \%$ between his analytic calculations and numerical estimates. In 2018 Chan carried out a more general comparison of numerical and analytic algorithms for estimating spacecraft collision probabilities [4]. Three analytic and three numerical methods were tested. The MECSA method was found to be best overall based on comparisons of both accuracy and computation time.

Wen and Gurtil (2016) developed the concept of the relative reachable domain [3]. This takes into account both the initial uncertainties in position and later uncertainties due to control maneuvers to described the potential relative positions of two objects. Under the assumptions of circular orbits analytic expressions for the geometry of the relative 
reachable domain can be calculated. The authors claim this improves on state of the art in collision risk assessment methods.

Recent research by Railand (2020) considers the more general question of how to set up a constellation of satellites so as to minimise collision probabilities [5]. Minimum Space Occupancy (MiSO) orbits are concluded to significantly reduce collision risk, however intensive numerical calculations are required to compute these orbits.

In our analytic work below, e.g. in section 4 we roughly follow the methods developed by Chan but with additional simplifications.

\section{Data Analysis}

\subsection{Case Study: Iridium Collison}

Iridium-33 was part of a 66 satellite constellation. The satellite distribution consisted of 6 orbital planes with 11 satellites placed equidistant on each. On the 10th February 2009 the $560 \mathrm{~kg}$ Iridium-33 satellite and $950 \mathrm{~kg}$ Kosmos-2251 collided at an altitude of $789 \mathrm{~km}$ and a speed of $11.7 \mathrm{~km} / \mathrm{s}$ in the first hypervelocity collision between two satellites [7]. Fig. 2 shows the orbits of the two bodies calculated from two line element set data before the crash.

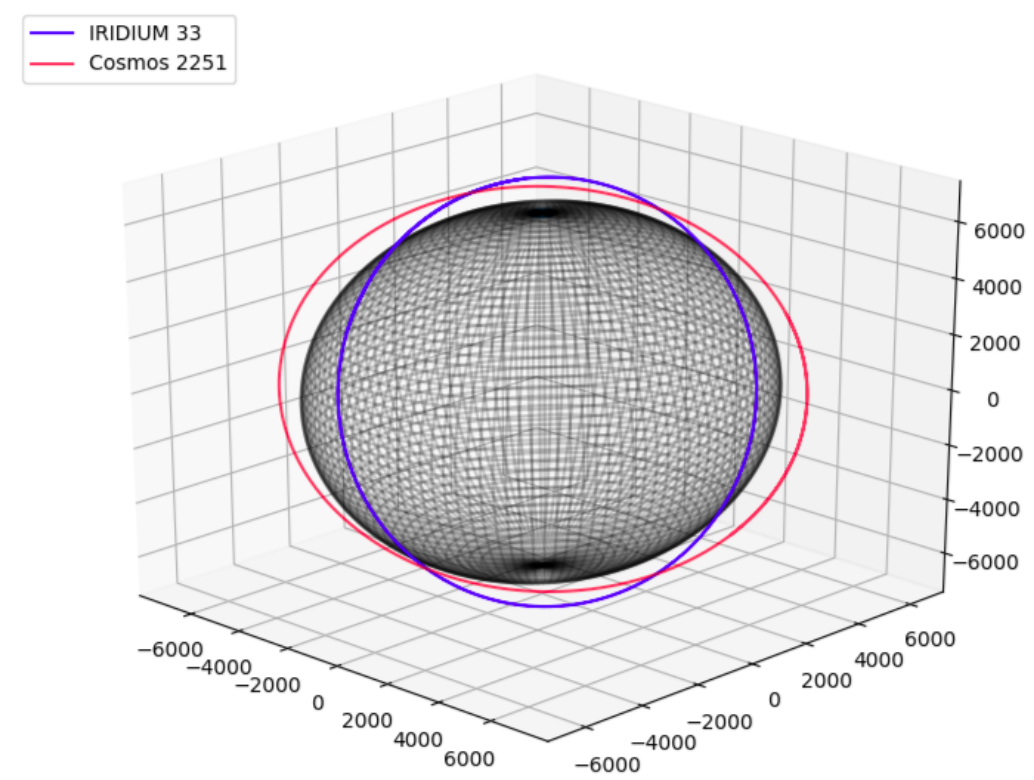

Figure 2. Iridium \& Kosmos orbits from latest TLE Data before the crash

T Kelso (maintainer of the www . celestrak. com database) used the Pentagon's publicly available data to estimate that two satellites should have missed each other by $584 \mathrm{~m}$ [6]. The Joint Space Operations Center (JspOC) provided daily conjunction reports, and Iridium got over 400 of these each week across the entire constellation, but there were 
complaints the error was too high to be useful [9]. A number of factors cause orbits to change making calculating collisions difficult. These include: accidental release of fuel, drag from the Earth's atmosphere, gravitational tugs from Sun/Moon/Earth. [6]

\subsection{Estimating Positional Uncertainty}

Historic data on two line element sets can be used to estimate uncertainties in satellite position. A historical dataset consisting of 1842 two line element sets was downloaded from the Celestrak website. These were transformed into a Cartesian coordinate system and projected onto the $y=0$ plane. The results of this calculation are shown in Fig. 3. Rough estimates of the uncertainties in position can then be made.

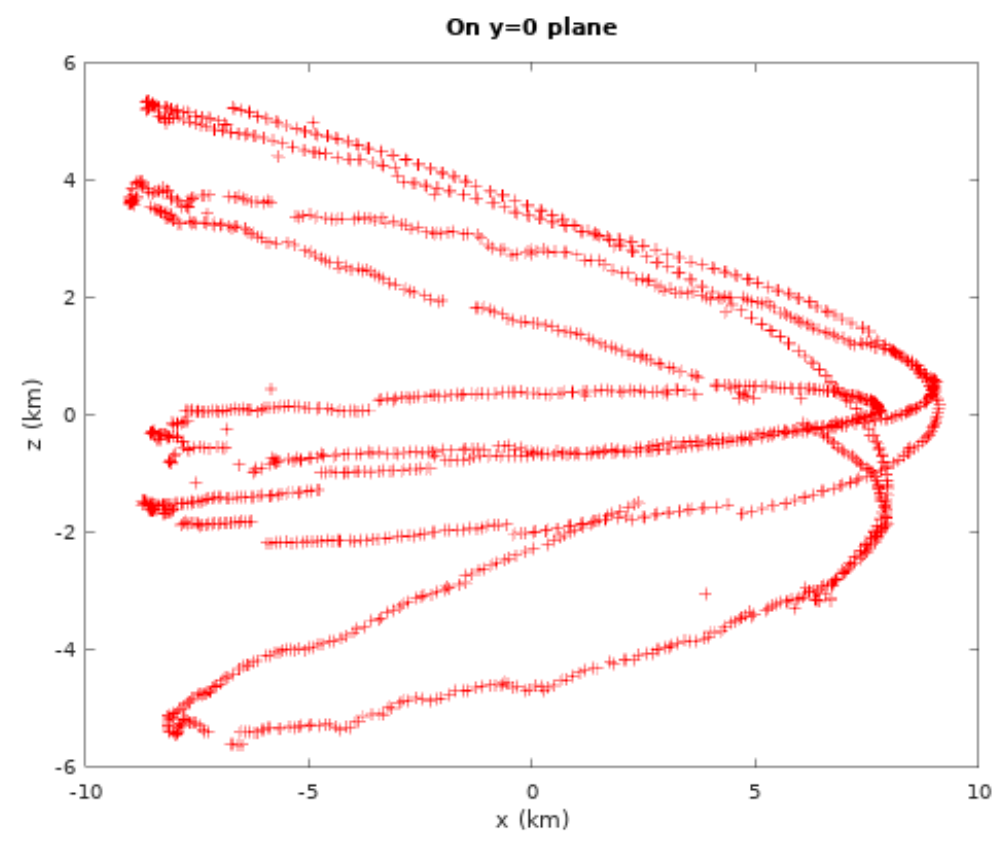

Figure 3. Historical data from NOAA-17 after coordinate transformation showing intersection of orbits with $y=0$ plane. $\sigma_{x} \approx 10 \mathrm{~km}$ and $\sigma_{z} \approx 6 \mathrm{~km}$

\section{A very simple collision model}

In this section we estimate the probability of a collision occurring between two satellites where the motion of both is completely uncorrelated and there is no active control of either. This is similar to the problem of finding the probability of two pieces of space junk colliding. We note that there are several theories that model the probability of collisions (see for instance Ref. [4] for a selection of numerical and analytical models). The integrals necessary to compute this are complicated and hence numerous approximations are made to simplify the calculations. Here we present a more ad hoc method which could be converted to a more formal asymptotic approach.. 
Firstly, we are interested in collisions where the paths intersect for only a short distance (rather than the problem of collisions where satellites are on nearly the same orbit and travelling at nearly the same speed). This is the situation illustrated in Fig. 1. Let us start by approximating the probability distribution of each satellite by assuming that it is equally likely to be anywhere on its orbit and that there are small variations in its path normal to the path direction. We also assume that during an 'encounter', when the paths get near, the satellites travel in straight lines.

Assuming the orbit is circular and of length $2 \pi R$ (where the distance from the earth's centre is $R$ ), we use coordinates for the first satellite of $R \theta$ for the distance along the path ( $0 \leq R \theta<2 \pi R$ is one orbit), $z$ for the radial coordinate (normal to the earth's surface) and $y$ for the coordinate normal to both $z$ and $R \theta$. For the simplest case, of a Gaussian distribution) the probability per unit volume of the satellite being at point $(R \theta, y, z)$ is given by

$$
P_{1}(R \theta, y, z)=\frac{1}{2 \pi R} \frac{1}{\pi \sigma_{1}^{2}} \exp \left(-\frac{z^{2}+y^{2}}{\sigma_{1}^{2}}\right)
$$

We now consider a second satellite travelling on a different orbit with mean height $H$ above satellite 1 and travelling at an angle $\phi$ (with slope $\alpha=\tan (\phi)$ ) to the first satellite. Then, locally to the encounter we can write (this is essentially the 'encounter coordinate system' described by Chan $[2,4]$. Hence since the first satellite has a mean position moving along the $R \theta$ axis (the line $y=0, z=0$ ) the second satellite will have a mean position that travels along the line $y=\alpha R \theta=a y, z=H$. The probability density function of this second satellite is given by

$$
P_{2}(R \theta, y, z)=\frac{1}{2 \pi(R+H)} \frac{1}{\pi \sigma_{2}^{2}} \exp \left[-\frac{(z-H)^{2}+(\cos \phi y-\sin \phi R \theta)^{2}}{\sigma_{2}^{2}}\right]
$$

Given these two probability distributions, we are now interested in determining the probability of the two satellites getting within a critical distance $R_{A}$. (Here $R_{A}$ is the sum of the radii of the two satellites which are assumed spherical). A formal definition of this probability could be calculated but it would involve considerations of three very different length scales namely are $R, \sigma$ and $R_{A}$. In practice we know that $R \gg \sigma \gg R_{A}$ and we wish to exploit this to find the probability approximately (note we have already exploited these disparate scales by making the different distributions tangential and normal to the orbit). One additional length scale is $H$ (the difference in the orbit heights) and the sensible distinguished limit to consider is where $H$ is similar in size to the uncertainty in position $\sigma_{1,2}$.

Our first observation is that, if the critical distance is small then the probability of the two satellites being within a distance $R_{A}$ is approximately $\left(4 \pi R_{A}^{3} / 3\right) P_{1}(R \theta, y, z) P_{2}(R \theta, y, z)$. The total probability of a collision during this encounter is then

$$
\int_{0}^{2 \pi} \int_{-\infty}^{\infty} \int_{-\infty}^{\infty} \frac{4 \pi R_{A}^{3}}{3} P_{1}(R \theta, y, z) P_{2}(R \theta, y, z) d z d y d(R \theta)
$$

We can now approximate this integral (formally we might use the method of steepest decent). Because the paths are at a reasonable angle $(\alpha=O(1)$ and $\alpha \neq 0)$ it follows 
that the joint probability decays in $\theta$ so that we can approximate this to

$$
\int_{-\infty}^{\infty} \int_{-\infty}^{\infty} \int_{-\infty}^{\infty} R_{A} P_{1}(R \theta, y, z) P_{2}(R \theta, y, z) d z d y d(R \theta)
$$

or

$$
\int_{-\infty}^{\infty} \int_{-\infty}^{\infty} \int_{-\infty}^{\infty} \frac{R_{A}^{3}}{3 \pi^{3} R^{2}} \frac{1}{\sigma_{1}^{2} \sigma_{2}^{2}} \exp \left[-\frac{z^{2}+y^{2}}{\sigma_{1}^{2}}-\frac{(z-H)^{2}+(y \cos \phi-x \sin \phi)^{2}}{\sigma_{2}^{2}}\right] d z d y d x
$$

Nondimensionalising this we get

$$
\frac{R_{A}^{3}}{3 \pi^{3} R^{2}} \frac{\sigma_{1}}{\sigma_{2}^{2}} \int_{-\infty}^{\infty} \int_{-\infty}^{\infty} \int_{-\infty}^{\infty} \exp \left[-\left(z^{2}+y^{2}\right)-\sigma^{2}\left((z-h)^{2}+(y \cos \phi-x \sin \phi)^{2}\right)\right] d z d y d x
$$

where we use the notation $\sigma=\sigma_{1} / \sigma_{2}$, and $h=H / \sigma_{1}$. Following the integration through the result is

$$
\frac{R_{A}^{3}}{3 \pi^{3 / 2} R^{2}} \frac{1}{\sqrt{\sigma_{1}^{2}+\sigma_{2}^{2}}} \frac{1}{\sin \phi} \exp \left[\frac{-h^{2}}{\sigma_{1}^{2}+\sigma_{2}^{2}}\right]
$$

This rule tells us that the probability of the of a single collision between a pair of satellites on different orbits scales:

- with the volume of the satellites i.e. with $R_{A}^{3}$,

- inversely with the volume of the spherical shell within which the satellites move scaling inversely with $R^{2} \sigma_{1,2}$,

- inversely with the sin of the angle between the paths.

There is also a very strong dependency on the mean distance the satellites travel above each other (Gaussian dependency on $H$ ). Note here that for a more general encounter $H$ represents the minimum of the expected distance between the satellites. Note for more general situations it is necessary to multiply this probability by two (because orbits intersect and create possible encounters twice per orbit). Furthermore, if an orbit contains many satellites the probability should be scaled by that number. Additionally the satellite may intersect several orbits of other satellites. Finally the angle at which the satellite encounters the path of other satellites may vary. For example if a polar orbit encounters satellites on a fixed azimuthal orbit (where the orbits will precess) an average probability over possible angles of encounter will need to be taken. Finally this probability is the probability of a collision by the satellite on a single orbit so this needs (roughly) multiplying by the expected number of orbits.

A plot of how the collision probability varies with $H$ is given in Fig. 4

\section{Probability of Collision with satellites in a densely packed orbit}

In this section, we consider the probability of collision when a satellite crosses another densely packed orbit containing $n$ satellites. 


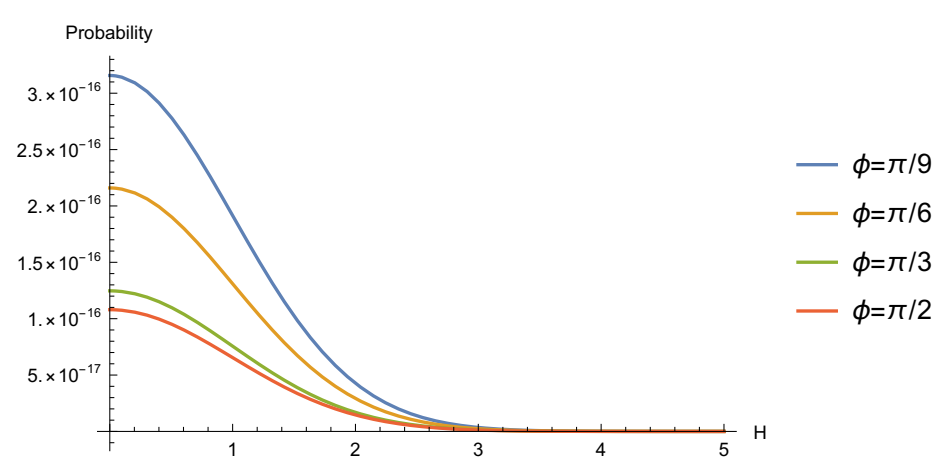

Figure 4. Collision probabilities as a function of $H$ for typical parameter values

\subsection{Probability of collision}

We predict probabilities of collision (rather than being assured of a collision) because of uncertainty in the satellites' trajectories which can arise, for example, from accidental release of fuel, drag from the Earth's atmosphere, gravitational tugs from Sun/Moon/Earth [6]. We use the probability of collision $p$ calculated by Chan [2]. At leading order this is:

$$
p=e^{-v / 2}\left(1-e^{-u / 2}\right)
$$

where

$$
\begin{gathered}
u=\frac{R_{A}^{2}}{\sigma_{x}^{\prime} \sigma_{z}^{\prime}}, \\
v=X_{e}^{2} \frac{1+\left[\left(\sigma_{z}^{\prime} / \sigma_{x}^{\prime}\right)^{2}-1\right]\left(\frac{X_{p}^{\prime 2}}{X_{p}^{\prime 2}+Z_{p}^{\prime 2}}\right)}{\sigma_{z}^{\prime 2}},
\end{gathered}
$$

where $X_{e}$ is the minimum pass distance between the satellites, $\sigma_{x}$ and $\sigma_{z}$ are the standard deviations of the satellite's paths in the two principal directions, $R_{A}$ is the combined cross sectional radius, $X_{p}$ and $Z_{p}$ are the nominal miss distances in the two principal directions.

Similar quantities are discussed in section 4 where a model for the collision probability is derived. We note here that the parameters can be calculated from satellite trajectory data. We also note that many of these quantities are in the principal direction and are transformed by rotation through an angle $\theta$. The parameters $X_{p}$ and $Z_{p}$ can then be expressed as

$$
\left(X_{p}^{\prime}, Z_{p}^{\prime}\right)=\left(X_{e} \cos (\theta), X_{e} \sin (\theta)\right)
$$

for

$$
\theta=\frac{1}{2} \tan ^{-1}\left(\frac{2 p_{x z} \tilde{\sigma}_{x} \tilde{\sigma}_{z}}{\tilde{\sigma}_{x}^{2}-\tilde{\sigma}_{z}^{2}}\right)
$$

for correlation coefficient $\rho_{x z}$, where tildes indicate that the standard deviations aren't in the principal directions. For a circular cross section $\theta= \pm \pi / 4$. We note that this means $v$ can be rewritten as

$$
v=X_{e}^{2} \frac{1+\left[\left(\sigma_{z}^{\prime} / \sigma_{x}^{\prime}\right)^{2}-1\right]\left(\frac{\cos ^{2}(\theta)}{\cos ^{2}(\theta)+\sin ^{2}(\theta)}\right)}{\sigma_{z}^{\prime 2}} .
$$

Therefore, we note that $v \sim X_{e}^{2}$. 
Table 1. Three examples of parameter values as taken from [2]. The parameters used for Fig. 6-8 are marked in the three corresponding colours. The parameter $X_{e}$ is marked in gray as this parameter is varied in those studies.

\begin{tabular}{ccccc}
\hline \hline Quantities & Case 1 & Case 2 & Case 3 \\
\hline \hline$R_{A}(\mathrm{~km})$ & 0.005 & 0.01 & 0.01 \\
$\sigma_{x}^{\prime}(\mathrm{km})$ & 0.05 & 10 & 3 \\
$\sigma_{z}^{\prime}(\mathrm{km})$ & 0.025 & 1 & 1 \\
$\theta$ & 0 & 0 & 0 \\
$X_{p}(\mathrm{~km})$ & 0.01 & 10 & 1 \\
$Z_{p}(\mathrm{~km})$ & 0 & 0 & 0 \\
$X_{e}(\mathrm{~km})$ & 0.01 & 10 & 1 \\
\hline
\end{tabular}

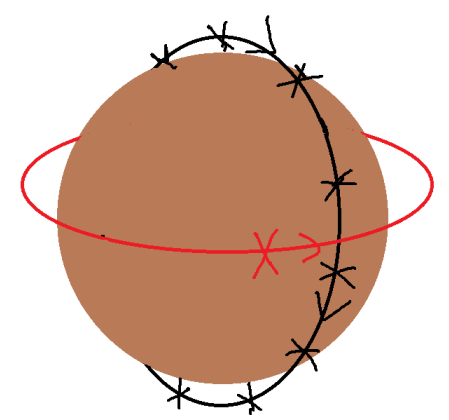

Figure 5. Diagram showing our satellite (in red) crossing another orbit of densely packed satellites in brown. Crosses indicate satellites and the arrows motion around the orbit.

In Table 1, three cases are listed with data taken from [2], showing the values of these parameters. These three cases provide the baseline values for our study in the next subsection, of how the collision probability changes as the density of satellites in the orbit we are crossing changes.

\subsection{Crossing a densely packed orbit}

In this section, we assume our satellite is crossing a densely packed orbit of satellites (see Fig. 5). We will consider how the probability of collision changes depending on the density of that orbit and the trajectory of our satellite.

If we vary the density of the satellites on the packed orbit, but keep other factors constant including type of satellite (so that $\sigma_{x}$ and $\sigma_{z}$ remain unchanged) and orbit angles (so that $\theta$ remains unchanged) then the only parameter changing will be $X_{e}$. (In fact $X_{p}$ and $Z_{p}$ will also vary as they are dependent on $X_{e}$ from Eq. 5.4). From Eq. 5.6 and Eq. 5.2, the probability of collision Eq. 5.1 remains unaffected.

Assuming all other parameters constant and $X_{e}$ varying then $v \sim X_{e}^{2}$ and $p \sim e^{-B X_{e}^{2}}$ for $B$ a constant. Therefore as the predicted distance between two satellites increases the probability they will collide decreases exponentially quickly as shown in Fig. 6. This fast 


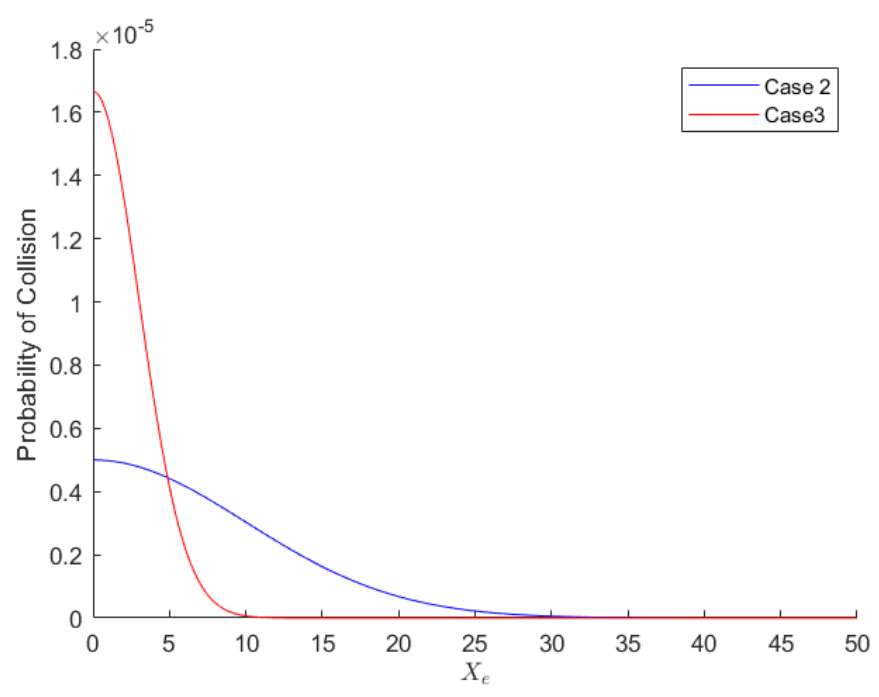

Figure 6. Decrease in probability of collision between two satellites as $X_{e}$ increases for two cases listed in Table 1.

decay also means that in most cases for collision we need only consider nearby satellites, or even just the nearest. This indicates that as the density of satellites increases, our concern is that we will need to pass other satellites more closely (so lower $X_{e}$ ), but our main concern is still the nearby satellites rather than those further away in the orbit.

We can make a simple estimate for how $X_{e}$ scales with $n$, the number of satellites on the densely packed orbit. We assume $n$ satellites are distributed evenly over the circular orbit at radius $R$ (where we take $R=6378+550=6928 \mathrm{~km}$ i.e. an orbit $550 \mathrm{~km}$ above the Earth of radius $6378 \mathrm{~km}$ ). Along the orbit the satellites are a distance of $2 \pi R / n$ apart (or $2 R \sin (\pi / n)$ in a straight line which are equal as $n \rightarrow \infty$ so assuming $X_{e} \ll R$ i.e $n \gg 1$ we will use the circular orbit approximation for distance between the satellites to define $X_{e}$ ). If we assume our satellite has its path chosen to maximise $X_{e}$ for the two satellites as it passes between i.e. it will pass equidistant between two satellites so $X_{e}=\pi R / n$. For this to be $<10 \mathrm{~km}$ you would need $\approx 2177$ satellites in the orbit.

In Fig. 7 we look at how the probability of collision increases as $n$ is increased. We assume we are passing between two satellites and we have a probability of colliding with each as $p_{1}$ and $p_{2}$ respectively, calculated using Eq. 5.1. We assume these two events are independent and take the total collision probability $p_{c o l}=1-\left(1-p_{1}\right)\left(1-p_{2}\right)$ i.e one minus the probability we don't collide with either (represented by crosses in Fig. 7). Assuming the chance of colliding with the satellite on either side are independent events is untrue as knowing our satellite is colliding or not colliding with one satellite would give information about our satellite's location thus changing the position distribution when calculating the likelihood of collision with the second satellite. However, we take this as a good initial assumption but further work would be required to consider how much the probability is different if the events are not assumed independent. In Fig. 7, we can see that the probability of collision increases as the number of satellites (density) in the crossed orbit increases. If we only consider the two nearest satellites (crosses), the 

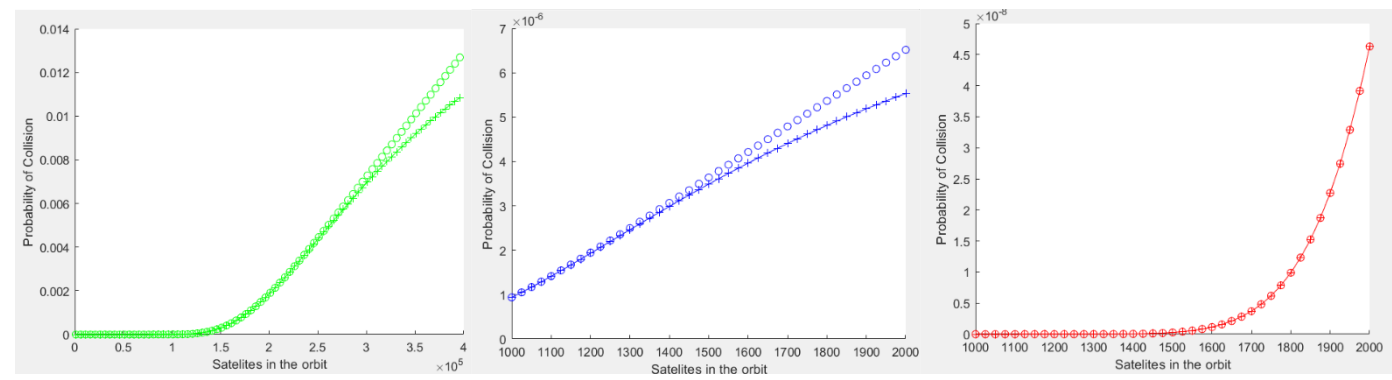

Figure 7. Change in probability of collision as our satellite passes through a uniformly distributed orbit of satellites, for increasing number of satellites in that orbit. We assume the satellite passes equidistant between two satellites in the orbit. Crosses account for probability of colliding with the two nearest satellites. Circles account for all satellites in the dense orbit which are within $50 \mathrm{~km}$ of our satellites orbit. The three graphs correspond to the three cases listed in Table 1 (Case 1 green, Case 2 blue, Case 3 red).

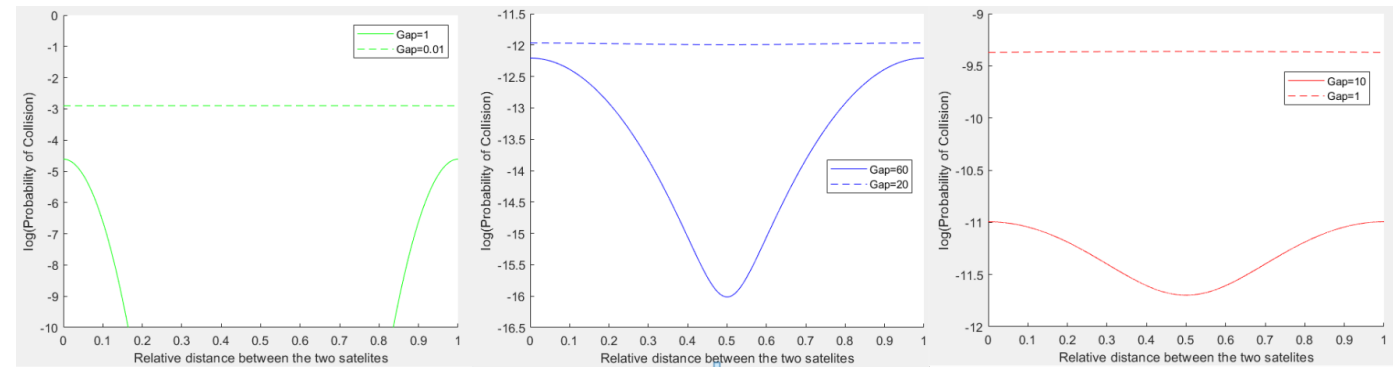

Figure 8. Change in probability of collision as the location of our satellite pass changes. We assume our satellite is passing between two satellites (which are part of a dense equally spaced orbit). The $x$-axis indicates position to pass between the satellites, with 0 and 1 representing a course straight through either of the closest satellites and 0.5 passing midway between them. Two sizes for the gap between satellites are indicated. The three graphs correspond to the three cases listed in Table 1 (Case 1 green, Case 2 blue, Case 3 red).

collision probability tends to a limit, which intuitively seems unrealistic. However, if we increase the number of satellites considered so we consider the satellites once removed at position $2 X_{e}$ away from us, taking $p_{c o l}=1-\left(1-p_{1}\right)\left(1-p_{2}\right)\left(1-p_{3}\right)\left(1-p_{4}\right)$ then this probability keeps increasing with higher density (circles in Fig. 7). The point at which including more than just the nearest pair of satellites becomes important is a function of the density of the satellites and also $\sigma_{x}$ and $\sigma_{z}$, likely reflecting these further away satellites only become important when there is a good probability their paths will overlap with our satellites. However, by this stage the standard deviation of satellites position may be large enough to indicate problems of collisions within its own constellation.

In Fig. 8 we consider the best position to pass between two satellites in a dense orbit. As would be expected, for larger miss distances $\left(X_{e}\right)$ and smaller satellite standard deviations, the probability of collision is least if you pass through the middle, as far from either satellite as possible. However, as $X_{e}$ is decreased the advantage of passing 
through the middle decreases, and any position choice has similar collision probabilities, even choosing a course straight towards another satellite (with $X_{e}=0$ ). This reflects the counter-intuitive optimal path if there is high uncertainty in the satellite position relative to distance between them so it may be slightly preferential to have a high probability of hitting one satellite as it means there is a much lower chance of hitting the second satellite, rather than having a medium change of hitting both satellites. Whether we consider just the two closest satellites or the next two or four beyond that in the orbit has little effect at the larger $X_{e}$ (solid lines in Fig. 8), where the middle path is best. However, it has a larger effect on the collision probability magnitude at smaller $X_{e}$ (dotted lines in Fig. 8) but the lines are a similar shape (horizontal) so we still observe minimal differences dependent on satellite course. This horizontal line reflects that if there is high uncertainty in satellite positioning relative to available pass distance, and a chance of collision is high everywhere then it doesn't make much difference what path is taken.

We have considered the probability of collision if two satellites pass within distance $X_{e}$, but there is the alternative question: What is the chance two satellites come within distance $X_{e}$ in the first place? If we have no information about the position of the two satellites, then we must assume they are equally likely to be at any position on each of their orbits. In this case the chance that the second satellite is within $X_{e}$ of the orbit overlap point (calculating distances along the orbit) when the first satellite is at the overlap point is $X_{e} /(\pi R)$. This is an initial estimate of the chance the two satellites come within distance $X_{e}$ at that intersection point, but, as the first satellite is moving too, this probability could be higher. There was insufficient time to obtain a better estimate which would be dependent on the angle $\theta$ between the two orbits, but this could be studied in further work. 


\section{Conclusions and further directions}

As orbit shells become crowded understanding how the probabilities of collisions between satellites on intersecting orbits becomes important. The Iridium-33 crash shows that this is more than a theoretical possibility. We showed that a simple formula for the probability of a collision can be obtained by exploiting small dimensionless numbers in the problem. We then extended our results to consider collisions involving orbits containing $n$ satellites. This included the counterintutive result that there are circumstances under which it is better not to aim to pass between the midpoint of two satellites to minimise the probability of a collision.

There are a number of ways in which the results could be extended. The single collision model was formulated in an ad hoc way, a more rigorous asymptotic formulation would be useful in particular allowing the errors introduced to be quantified.

In considering orbits containing multiple satellites we considered our satellite to be passing a single orbit of equally spaced satellites. Any real constellation will contain multiple orbits. Thus, an initial extension would be to calculate the chance of our satellite colliding during one full rotation of the Earth when it has to cross multiple orbits, and including both intersection points of those orbits. We have assumed it is possible to set up our satellite path to pass through the orbit to maximise the distance between our satellite and those in the orbit. Even if this were possible when crossing one orbit of satellites, this would not necessarily be possible when crossing multiple packed orbits without regular realignment. Thus, it would be interesting to see how this effect increases the collision probability during one Earth rotation, and how the phase difference of each orbit relative to each other affects that collision probability. As a first step the interaction with a known constellation such as the Iridium constellation could be analysed.

This information could then be used to consider interactions with the unknown layout of the, for instance, Space-X constellation. This could be done by first considering the probability of collision for a best-guess layout for the Space-X constellation. Then this could be extended to an optimisation problem with the probability of collision during one rotation as the cost function, to consider layouts of the constellation which lead to the smallest probability of collision.

\section{Acknowledgements}

ESGI162 was organised with support from representatives from: The Virtual Forum for Knowledge Exchange in the Mathematical Sciences (V-KEMS), The University of Huddersfield, The University of Bristol, and The University of Bath. V-KEMS consists of Knowledge Transfer Network (KTN), Isaac Newton Institute (INI), Newton Gateway to Mathematics, and International Centre for Mathematical Sciences (ICMS). The organisers are grateful to Overleaf for sponsoring the Study Group with free access to professional accounts 


\section{References}

[1] Campbell, M.E., 2004. Collision monitoring within satellite clusters. IEEE Transactions on Control Systems Technology, 13(1), pp.42-55.

[2] Ken Chan. International Space Station Collision Probability (2009). The aerospace corporation

[3] Wen, C. and Gurfil, P., 2016. Relative reachable domain for spacecraft with initial state uncertainties. Journal of Guidance, Control, and Dynamics, 39(3), pp.462-473.

[4] Chan, F.K., 2018, July. Comparison of Methods for Spacecraft Collision Probability Computations. In International Space Conference of Pacific-basin Societies (ISCOPS), Montreal, Canada.

[5] Reiland, N., Rosengren, A.J., Malhotra, R. and Bombardelli, C., 2020. Assessing and Minimizing Collisions in Satellite Mega-Constellations. arXiv preprint arXiv:2002.00430.

[6] New Scientist Satellite crash prediction is plagued with uncertainty.

[7] Wikipedia 2009 Satellite Collision.

[8] NASA Technical Reports (2009) The Collision of Iridium 33 and Cosmos 2251: The Shape of Things to Come PDF.

[9] Wikibooks Iridium 33 and Kosmos 2251.

\section{Appendix A Radial distance calculations between two normal RV's in $R^{3}$}

Let us suppose that we have two objects $X$ and $Y$ and at a particular time point $t$ with some uncertainty related to their locations, i.e. $X_{t} \sim N\left(\mu_{t}^{X}, \Sigma_{t}^{X}\right)$ and $Y_{t} \sim N\left(\mu_{t}^{Y}, \Sigma_{t}^{Y}\right)$. The (squared) euclidean distance between these two objects at time $t$ is a random variable $|Z|^{2}=|X-Y|^{2}$ where $Z \sim N\left(\mu_{t}^{X}-\mu_{t}^{Y}, \Sigma_{t}^{X}+\Sigma_{t}^{Y}\right)$. The probability density function of $|Z|^{2}$ is known to be non-standard but it can be seen as a linear combination of the noncentral chi-square distributions. There has been a lot of work done in the stats literature about these distributions. One quick method for evaluating such probabilities is based on the Saddle-Point Approximations for tail probabilities.

So in principle for each pair of objects at time $t$, once the locations' uncertainty is quantified by some Normals $N\left(\mu_{t}, \Sigma_{t}\right)$, one can quantify the probability of these two objects being, say, $\tau$ units apart:

$$
P\left(\left|Z_{t}\right|^{2}<\tau\right)=P_{X, Y}(\tau, t)
$$

The possible values of $\tau$ could be specified by the user which correspond to the $R_{A}$ term above or $r_{a}$ in Chan's paper [2].

If we have say three objects $X, Y$ and $W$, we could find at a given time $t$, the probability of a possible collision event as

$$
\min \left(P_{X, Y}(\tau, t), P_{X, W}(\tau, t), P_{W, Y}(\tau, t)\right)
$$

and so it can be generalised even further.

We show now how one could calculate the near miss event probability as $P\left(Z_{t}^{2}<\tau\right)$, for small $\tau$. Let's drop time $t$ subscript for a moment.

Note that $Z \sim N\left(\mu^{X}-\mu^{Y}, \Sigma^{X}+\Sigma^{Y}\right)$. Let $\Sigma=\Sigma^{X}+\Sigma^{Y}=O^{t} \Delta O$, where $O$ is the 
orthogonal matrix of the spectral decomposition. So $L=O Z \sim N\left(O \mu^{X}-O \mu^{Y}, \Delta\right)$, $\Delta=\operatorname{diag}\left(\delta_{1}^{2}, \delta_{2}^{2}, \delta_{3}^{2}\right)$. Then, $P\left(\left|Z^{2}\right|<\tau\right)=P\left(\left|L^{2}\right|<\tau\right)$ where $L$ is three dimensional such that $L_{i}$ are now independently normally distributed with mean $\left(O \mu^{X}-O \mu^{Y}\right)_{i}=\xi_{i}$ and variance $\delta_{i}^{2}$. So $|L|^{2}=L_{1}^{2}+L_{2}^{2}+L_{3}^{2}=\left(\xi_{1}+\delta_{1} Z_{1}\right)^{2}+\left(\xi_{2}+\delta_{2} Z_{2}\right)^{2}+\left(\xi_{3}+\delta_{3} Z_{3}\right)^{2}$ where $Z_{1}, Z_{2}$ and $Z_{3}$ are i.i.d $N(0,1)$. In particular, $\left(\xi_{i}+\delta_{i} Z_{i}\right)^{2}=\delta_{i}^{2}\left(\frac{\xi_{i}}{\delta_{i}}+Z_{i}\right)^{2} \sim \delta_{i}^{2} \chi_{1}^{2}\left(\frac{\xi_{i}^{2}}{\delta_{i}^{2}}\right)$, where $\chi_{1}^{2}(x)$ is the non-central chi square distribution with non-centrality $x$. In our case then the square euclidean distance between two Gaussian distributed Rv's X and Y is

$$
|L|^{2} \sim \delta_{1}^{2} \chi_{1}^{2}\left(\frac{\xi_{1}^{2}}{\delta_{2}^{2}}\right)+\delta_{2}^{2} \chi_{1}^{2}\left(\frac{\xi_{2}^{2}}{\delta_{2}^{2}}\right)+\delta_{3}^{2} \chi_{1}^{2}\left(\frac{\xi_{3}^{2}}{\delta_{3}^{2}}\right)
$$

which is a linear combination of non-central chi-squares.

\section{A.1 Integral form of the CDF of $|L|$}

So for evaluating the probabilities of the type $P(|L|<\tau)$ we will need to consider

$$
P(|L|<\tau)=\int_{L_{1}^{2}+L_{2}^{2}+L_{3}^{2}<\tau} \frac{1}{(2 \pi)^{3 / 2} \delta_{1} \delta_{2} \delta_{3}} \exp \left\{-\frac{1}{2} \sum_{i=1}^{3} \frac{\left(l_{i}-\xi_{i}\right)^{2}}{\delta_{i}^{2}}\right\} d l_{1} d l_{2} d l_{3}
$$

Applying the polar coordinates for $L=R v$, where $v$ is a spherical vector. The Lebesgue measure is therefore as $d l_{1} d l_{2} d l_{3}=R^{2} d R d v$, where $d v$ is the uniform measure on the ordinary sphere embedded in $R^{3}$.

We can write in these new coordinates the previous probability as

$$
P(|L|<\tau)=P(R<\tau)=\int_{R<\tau} d R \int_{S^{2}} d v \quad \frac{1}{(2 \pi)^{3 / 2} \delta_{1} \delta_{2} \delta_{3}} \exp \left\{-\frac{1}{2} \sum_{i=1}^{3} \frac{\left(R v_{i}-\xi_{i}\right)^{2}}{\delta_{i}^{2}}\right\} R^{2}
$$

These two expressions correspond to the same probability of Eq (2) in Chan's paper. Due to its complexity Chan considers the simplified 2-dimensional version which is shown in the expressions of Eq. 3-4 in that paper. Note that the mean of the Gaussian distribution there is $(0,0)$ which in our case it corresponds to non-centrality terms $\xi_{i}=0$. The problem is essentially of the same nature is the one shown above but just in two dimensions. The corresponding expressions are below:

\section{A.2 Two dimensional approximation}

In this case the directional vector $v$ is 2 dimensional. A parametrisation as $v_{1}=\sin (\theta)$ and $\left.v_{2}=\cos (\theta)\right)$ implies $d l_{1} d l_{2}=R d R d v=R \cos (\theta) d R d \theta$ : so

$$
\begin{gathered}
P(R<\tau)=\int_{R<\tau} d R \int_{S^{1}} d v \frac{1}{(2 \pi)^{2 / 2} \delta_{1} \delta_{2}} \exp \left\{-\frac{1}{2} \sum_{i=1}^{2} \frac{\left(R v_{i}-\xi_{i}\right)^{2}}{\delta_{i}^{2}}\right\} R \\
P(R<\tau)=\int_{R<\tau} d R \int_{S^{1}} d \theta \frac{1}{(2 \pi)^{2 / 2} \delta_{1} \delta_{2}} \exp \left\{-\frac{\left(R \sin (\theta)-\xi_{1}\right)^{2}}{2 \delta_{1}^{2}}-\frac{\left(R \cos (\theta)-\xi_{2}\right)^{2}}{2 \delta_{2}^{2}}\right\} R \\
P(R<\tau)=\int_{R<\tau} d R \int_{0}^{2 \pi} d \theta \frac{1}{(2 \pi)^{2 / 2} \delta_{1} \delta_{2}} \exp \left\{\left\{-\frac{\left(R \sin (\theta)-\xi_{1}\right)^{2}}{2 \delta_{1}^{2}}-\frac{\left(R \cos (\theta)-\xi_{2}\right)^{2}}{2 \delta_{2}^{2}}\right\} R\right.
\end{gathered}
$$


in the calculations above $\xi$ 's are the expected difference of locations at a given time $t$ which in Chan's paper (eqn 10) are simply 0. Our expression corresponds to the probability in Eq. 10 of Chan's paper. (Note that we have done the transformation of the principal axes already while diagonalising the Covarianace matrix $\Sigma=\Sigma_{X}+\Sigma_{Y}$ ).

In the central case of $\xi=0$, we have

$$
\begin{gathered}
P(R<\tau)=\frac{1}{2 \pi \delta_{1} \delta_{2}} \int_{R<\tau} R d R \int_{0}^{2 \pi} d \theta \exp \left\{\frac{-R^{2}}{2}\left(\frac{\sin \theta^{2}}{\delta_{1}^{2}}+\frac{\cos \theta^{2}}{\delta_{2}^{2}}\right)\right\} \\
\frac{\sin ^{2} \theta}{\delta_{1}^{2}}+\frac{\cos ^{2} \theta}{\delta_{2}^{2}}=\frac{1}{\delta_{1}^{2}}+\left(\frac{1}{\delta_{1}^{2}}-\frac{1}{\delta_{2}^{2}}\right) \cos ^{2} \theta=\frac{1}{\delta_{1}^{2}}+\left(\frac{1}{\delta_{2}^{2}}-\frac{1}{\delta_{1}^{2}}\right) \frac{(1-\cos 2 \theta)}{2}=\frac{1}{2 \delta_{1}^{2}}+\frac{1}{2 \delta_{2}^{2}}-\frac{(\cos 2 \theta)}{2 \delta_{2}^{2}}
\end{gathered}
$$

and so

$$
\exp \left\{\frac{-R^{2}}{2}\left(\frac{\sin \theta^{2}}{\delta_{1}^{2}}+\frac{\cos \theta^{2}}{\delta_{2}^{2}}\right)\right\}=\exp \left\{\frac{-R^{2}}{2}\left(\frac{1}{2 \delta_{1}^{2}}+\frac{1}{2 \delta_{2}^{2}}\right)+\frac{R^{2}}{2} \frac{\cos 2 \theta}{\delta_{2}^{2}}\right\}
$$

implying that

$$
\begin{aligned}
P(R<\tau)= & \frac{1}{2 \pi \delta_{1} \delta_{2}} \int_{R<\tau} R \exp \left\{\frac{-R^{2}}{2}\left(\frac{1}{2 \delta_{1}^{2}}+\frac{1}{2 \delta_{2}^{2}}\right)\right\} d R \int_{0}^{2 \pi} \exp \left\{\frac{R^{2}}{2} \frac{\cos 2 \theta}{\delta_{2}^{2}}\right\} d \theta \\
P(R<\tau)= & \frac{1}{2 \pi \delta_{1} \delta_{2}} \int_{R<\tau} R \exp \left\{\frac{-R^{2}}{2}\left(\frac{1}{2 \delta_{1}^{2}}+\frac{1}{2 \delta_{2}^{2}}\right)\right\} d R 4 \int_{0}^{\pi} \exp \left\{\frac{R^{2}}{2} \frac{\cos \theta}{\delta_{2}^{2}}\right\} d \theta \\
& =\frac{4}{2 \pi \delta_{1} \delta_{2}} \int_{R<\tau} R \exp \left\{\frac{-R^{2}}{2}\left(\frac{1}{2 \delta_{1}^{2}}+\frac{1}{2 \delta_{2}^{2}}\right)\right\} I_{0}\left(\frac{R^{2}}{2 \delta_{2}^{2}}\right) d R
\end{aligned}
$$

where $I_{0}$ is the modified Bessel function of the first kind. This result does not look too different from that of Chan in Eq. 22. 